\title{
Review: Amoxycillin and folate inhibitors are as effective as newer, more expensive antibiotics for acute sinusitis
}

de Ferranti SD, Ioannidis JP, Lau J, dnninger WV, Barza M.Are anoxycillin and folate inhibitors as effective as other antibiotics for acute sinusitis? A neta-analysis. BMJ. 1998 Sep 5;317: $632-7$

\section{Questions}

In patents with acute sinusitis, what is the effectiveness of antibiotics on the natural course of disease? What is the effectiveness of amoxycillin or folate inhibitors compared with newer, more expensive antibiotics?

\section{Data sources}

Stidies were identified in MEDLNE (1966 to May 1998) using terms for antbiotic classes and sinusitis; hand searches were done in Excerpta Medica, abstracts of the interscience conference on antimicrobial agents and chemotherapy (1993 to 1997); and references of trials, reviews, and special issues were reviewed.

\section{Sludy selection}

Studies were selected if they were randomised controlled trials that compared amoxycillin or a folate inhibitor with another broad-spectrum antibiotic or compared antibiotics with placebo in patients with acute sinusitis or an acute exacerbation of chronic sinusitis. Studies were excluded if they were of subacute or chronic sinusitis or compared doses of nonantimicrobial drugs.

\section{Commentary}

This review by de Ferranti and colleagues confirms that antibiotics provide a somewhat greater benefit for acute sinusitis than does placebo: 7 patients need to be treated with any antibioric to "cure" one additional patient within 48 hours. Most patients (69\%) recovered spontaneously, even without antibiotics.

In this review, the outcomes measured include both signs and symptoms, which are not defined clearly enough to allow us to judge their clinical relevance. Furthermore, the rials may generally represent only severely ill patients. Diagnosis depended on radiographic evidence or culture of sinus aspirations for some patients. One sudy that

Data extraction

Data were extracted independently by 2 investigators on study and patient characteristics, drug type, diagnosis of sinusitis, outcome (cinical cure, improvement, and failure within $48 \mathrm{~h}$ of the end of treat ment), and methodological quality.

\section{Main results}

27 studies (2717 patients) were included. In 6 placebo-controlled trials, fewer patients who received any antibiotic had clinical failure than did patients who received placebo $\{P=0.007\}^{*}$ (Table). No difference in clinical failure existed between new antibiotics and amoxycillin in 13 trials $\{P=0.47\}^{*}$ (Table) or between new antibiotics and folate inhibitors in 8 trials $\{P=0.86\}^{*}$ (Table). Antibiotics were more effective than placebo in achieving clinical cure (relative risk [RR] $1.33,95 \%$ CI 1.02 to

Any antibiotic vs placebo and new antibiotics vs amoxycillin or folate inhibitors for acute sinusitis 48 hours after end of treatment $†$

\begin{tabular}{|c|c|c|c|c|}
\hline \multirow[t]{2}{*}{ Outcome } & \multicolumn{2}{|c|}{ Weighted event rates } & \multirow[t]{2}{*}{$\operatorname{RRR}(95 \% \mathrm{CD})$} & \multirow[t]{2}{*}{$\mathrm{NNT}(\mathrm{CI})$} \\
\hline & Any antibiotic & Placebo & & \\
\hline \multirow{5}{*}{$\begin{array}{r}\text { Clinical } \\
\text { failure }\end{array}$} & $13 \%$ & $28 \%$ & $46 \%(21$ to 63$)$ & $7(4$ to 26$)$ \\
\hline & New antibiotics & Amoxycillin & & \\
\hline & $8.6 \%$ & $9.5 \%$ & $14 \%(-19$ to 38$)$ & Not significant \\
\hline & New antibiotics & Folate inhibitors & RRI (CI) & $\mathrm{NNH}$ \\
\hline & $13.3 \%$ & $12.7 \%$ & $1 \%(-48$ to 97$)$ & Not significant \\
\hline
\end{tabular}

tAbbreviations defined in Glossary; RRR, NNT, and CI calculated from data supplied by author.

used inclusion criteria used by most family doctors (sinusitis-like symptoms) showed no beneft from antibiotics ( $85 \%$ of both control-and antibiotic-group patients recovered in 10 days).

In addition, the study shows that modem antibiotics (co-amoxiclav, pivampicillin, azithronitycin, clarithromycin, roxithromycin, cefaclor, cefixime, cefpodoxime, cefuroxime, cephalexin, minocycine, doxycycline, of tetracycline) provide no advantage over traditional ones (amoxycillin, co-trimoxazole, trimethoprim plus sulfametopyrazine, or brodimoprim). No serious complications were reported among 2717 patients.

One might therefore conclude that anti-
1.74), but new antibiotics were not more effective than amoxycillin (RR 1.04, CI 0.98 to 1.11 ) or a folate inhibitor (RR 1.01, Cr 0.88 to 1.17 ).

\section{Canclusions}

In patients with acute sinusitis, any antibiotic reduces clinical failure. Newer, more expensive antibiotics are not superiot to amoxycillin or folate inhibitors.

Sotwes of funding: Agenoy for Health Care Policy and Research and National lnstitute of Allergy and infections Diseases.

For correspondence: Di. 7. Lau, Division of Chinical Care Reseawh, Deportment of Medianne, New England Medial Conter; 750 Wasbington Street, Boston, MA 02111, USA. FAX 617-636-8023.

*P values calculated from data supplied by author: biotics and the traditional options are no less effective than more modern or expensive options) provide modest benefit for patients with acute sinusitis. It is less clear whether any benefit exists for patients with less well-defined acute sinusitis. Some patients will elect to use antibiotics to shorten their illiness rather than do nothing.

Cbristopher Del Mar; MB, BChi, MA University of Queensland Herstom, Queensland, Australia 